\title{
Sobre la estructura sintagmática de la aposición explicativa*
}

\author{
Carlos Muñoz Pérez ${ }^{* *}$ \\ Universidad de Buenos Aires, Argentina
}

\begin{abstract}
Resumen
El presente trabajo pretende evaluar la adecuación de dos tipos de análisis de las estructuras apositivas explicativas: los análisis simétricos y los análisis asimétricos. Asumiendo como marco teórico la gramática generativa contemporánea, se muestra cómo los análisis simétricos requieren tomar supuestos fuertes con respecto a la naturaleza de la sintaxis. Por tanto, se presenta un análisis asimétrico basado en una relación simétrica subyacente. Esta propuesta permite salvar los problemas conceptuales del análisis simétrico, a la vez que permite explicar (i) la naturaleza predicativa de la aposición explicativa, (ii) su carácter speaker-oriented, (iii) la condición de adyacencia entre ambas partes de la aposición, (iv) la presencia de adverbios entre ambas partes de la aposición, y (v) el alcance dispar de cuantificadores en ambos términos de la aposición.
\end{abstract}

\footnotetext{
* Una versión preliminar de este trabajo fue expuesta en las Primeras Jornadas Patagónicas de Lingüística Formal en octubre de 2011. Agradezco a todas las personas que se interesaron en aquel momento por la temática de mi comunicación y que hicieron preguntas y observaciones. Esta investigación se benefició de mi participación en el proyecto UBACyT 20020100100014, "Léxico y gramática: una perspectiva teórico-aplicada y su extensión a otras áreas". Los comentarios realizados sobre un manuscrito por Hilda Albano, Daniel Romero, Mabel Giammatteo y Augusto Trombetta resultaron de suma importancia para llegar a esta versión final. Todos los posibles errores, por supuesto, son de mi entera responsabilidad.

** Para correspondencia dirigirse a: Carlos Muñoz Pérez (cmunozperez@filo.uba.ar), Instituto de Lingüística, Universidad de Buenos Aires (UBA), 25 de Mayo 217, primer piso (CP 1002), Ciudad Autónoma de Buenos Aires, Argentina.
} 
Palabras clave: gramática generativa, aposición explicativa, ensamble simétrico, etiquetamiento.

\title{
ON THE PHRASE STRUCTURE OF THE EXPLICATIVE APPOSITION
}

\begin{abstract}
The present paper aims to evaluate the adequacy of two types of analysis of appositive constructions: the symmetric and asymmetric analysis. Grounded in contemporary generative grammar, it is shown that the symmetric analysis requires taking several assumptions about the nature of syntax. Thus, an asymmetric analysis is presented based on an underlying symmetric relation between both parts of the apposition. This proposal neutralizes the conceptual problems of a symmetric approach and, at the same time, permits to explain (i) the predicative nature of explicative appositions, (ii) its speakeroriented character, (iii) the adjacency condition between both parts of appositive constructions, (iv) the presence of adverbs between both parts of the apposition, and (v) the difference on scope relations between quantifiers on both parts of the apposition.
\end{abstract}

Key words: generative grammar, explicative apposition, symmetrical merge, labeling.

Recibido: $15 / 04 / 2012 \quad$ Aceptado: $10 / 10 / 2012$

\section{INTRODUCCIÓN}

Las aposiciones explicativas suelen ser una de las construcciones sintácticas que los estudiantes de gramática reconocen con mayor facilidad. Quizá esto se deba a que poseen ciertas características bien definidas que, en conjunto, no comparten con otras construcciones:

(1) Propiedades salientes de la aposición explicativa

a. sus constituyentes son dos sintagmas de determinante ${ }^{1}$ correferenciales.

1 Angela Di Tullio (c.p.) señala que no parece haber motivos teóricos o empíricos para no incluir en la denominación de aposiciones ciertas construcciones en las que se yuxtaponen 
b. constituto y constituyentes tienen la misma distribución sintáctica.

c. la relación entre ambos constituyentes es predicativa, parentética ${ }^{2}$ (no restrictiva) y no-argumental.

d. poseen una marcada entonación prosódica descendente (que se transcribe sistemáticamente a través de comas).

En la descripción, ambas partes de la aposición suelen ser analizadas como constituyentes que se encuentran en un mismo nivel jerárquico estructural. Así, un análisis sintáctico habitual para estas estructuras es el ejemplificado en (2). Éste suele ser considerado el análisis "más simple" para la aposición explicativa.

(2) [[Tus hermanos], [los peores vecinos que tuve]], deben cien pesos.

En el nivel teórico, esta descripción se relaciona con el análisis propuesto por Hockett (1958: 186), quien supone que en la aposición “....ambos constituyentes son [...] atributos, lo que equivale a decir que cada uno es al mismo tiempo atributo del otro y núcleo con respecto al otro". Esto, en términos más actuales, equivale a decir que existe una relación de mando-c mutua entre ambos constituyentes de la aposición ${ }^{3}$. La estructura a la que me refiero es esquematizada en (3), en donde la etiqueta $\mathrm{SD}_{\mathrm{Apos}}$ señala la construcción apositiva, mientras que $\mathrm{SD}_{1}$ y $\mathrm{SD}_{2}$ señalan cada uno de los términos de la aposición.

dos sintagmas de naturaleza no nominal (e.g., [[Aqui] [arriba]], o [Dijo [[eso], [que iba a venir]]). De momento, dejo esta cuestión de lado para centrarme en las aposiciones nominales (explicativas).

2 Con "interpretación parentética" me refiero, por ejemplo, a la posibilidad de poder parafrasear la oración de (2) en términos de una cláusula relativa apositiva (i) o de una construcción parentética (ii).

(i) Tus hermanos, que son los peores vecinos que tuve, deben cien pesos.

(ii) Tus hermanos -son los peores vecinos que tuve- deben cien pesos.

3 Existen muchos análisis que se diferencian del de Hockett al considerar que el segundo término de la aposición es un modificador del primero (e.g., Di Tullio 1997; Kovacci 1990). Sin embargo, dado que en estos análisis persiste la relación de mando-c mutuo entre ambas partes de la aposición, consideraré este tipo de análisis como variantes notacionales del análisis de Hockett. 
(3)

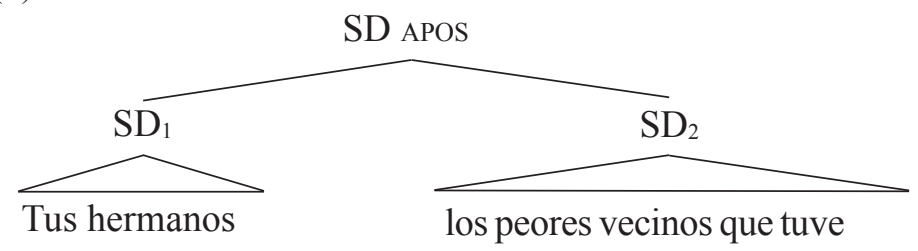

Una alternativa lógica a este tipo de análisis, aunque generalmente considerada más compleja, consistiría en asumir que existe una relación de tipo asimétrico entre ambos SSDD (es decir, solo uno de ellos mandaría-c al otro). Existen varias maneras de proponer un análisis "asimétrico" de la aposición, dependiendo de la estructura y longitud del $p a t h^{4}$ que se asuma entre ambos SSDD. Un esquema de este tipo de derivación se brinda en (4), en donde los puntos suspensivos indican que la cantidad de estructura que media entre ambos SSDD puede variar de acuerdo a cada análisis particular.

(4)

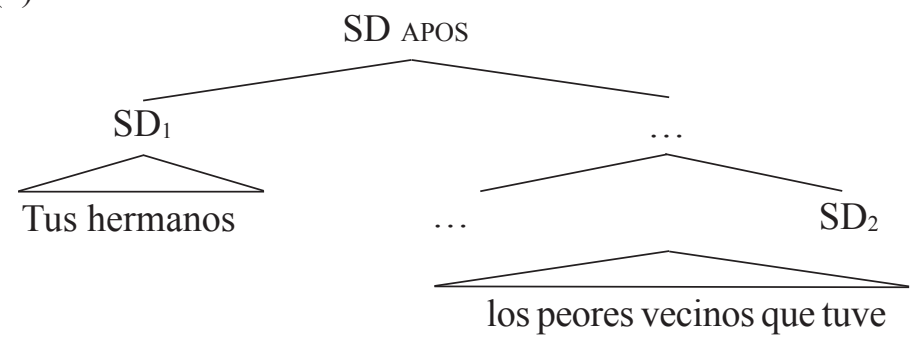

El presente trabajo tiene como objetivo evaluar las posibilidades técnicas de ambos tipos de análisis a partir de un modelo teórico generativo; más puntualmente, el sistema basado en la operación Agree (Chomsky 2001). Se pretende demostrar que, más allá de las apariencias, un análisis asimétrico de la aposición explicativa resulta mucho más simple en términos conceptuales que uno simétrico, además de ser empíricamente más adecuado.

En el apartado 2, se discuten los requerimientos conceptuales y las ventajas empíricas de un análisis de la aposición explicativa basado en una relación de mando-c simétrico entre ambos SSDD. En el apartado 3,

4 Tomo la definición ofrecida por Hornstein (2010: 37), según la cual un path es la distancia entre dos elementos en la estructura sintáctica definida a partir del número de nodos que hay entre ambos. 
se propone un análisis asimétrico de la aposición explicativa basado en el movimiento de uno de los términos desde una configuración estructural simétrica subyacente. Esto permitiría derivar todas las predicciones empíricas necesarias para una correcta descripción de la construcción y, a la vez, libraría el análisis de varias estipulaciones necesarias en un análisis puramente simétrico. El apartado 4 contiene las conclusiones del trabajo.

\section{SOBRE LOS REQUERIMIENTOS TÉCNICOS DEL ANÁLISIS SIMÉTRICO DE LA APOSICIÓN EXPLICATIVA}

El primer paso al intentar evaluar un análisis sintáctico particular es verificar si el modelo teórico que se asume permite postular tal análisis. Como se mencionó en la introducción, Hockett (1958) caracteriza la aposición como una especie de construcción de doble núcleo, en la que ambos SSDD están en una relación de yuxtaposición que los pone en el mismo nivel estructural. Esta configuración es la que se obtiene si se ensamblan dos SSDD, según el esquema de (5).

(5)<smiles>[18O]=C[Mg]</smiles>

Los signos de interrogación en (5) hacen referencia a un problema frecuentemente señalado en la bibliografía con respecto al ensamble de dos proyecciones máximas SX. Por definición, la operación Ensamble (Chomsky 1994) involucra la proyección de uno de los objetos sintácticos que son combinados. El patrón general observado es que un núcleo categorial $\mathrm{X}^{0}$ selecciona una proyección máxima SY y proyecta su tipo categorial al nuevo constituyente formado: $\left.{ }_{\mathrm{SX}} \mathrm{X}^{0} \mathrm{SY}\right]$. Esto generalmente se formaliza como un algoritmo, del cual cito un ejemplo en (6).

(6) Algoritmo de etiquetamiento (adaptado de Chomsky 2008: 145) ${ }^{5}$ En un ensamble $\{\alpha, \beta\}$, si $\alpha$ es un núcleo $X^{0}, \alpha$ proyecta.

\footnotetext{
5 Este algoritmo consta, en realidad, de dos partes. La segunda parte, que es la omitida aquí, refiere a casos en los que hay Ensamble Interno (i.e. movimiento) y, por tanto, no concierne a esta discusión.
} 
Ahora bien, el problema que involucra la estructura de (5) es que ambos objetos sintácticos ensamblados son proyecciones máximas. Por tanto, un algoritmo como el de (6) no puede determinar el tipo categorial de (5). Por supuesto, si bien parece "obvio" que la etiqueta correspondiente a (5) debería ser $S D$, nuestro interés teórico debe centrarse en explicar cómo es que la sintaxis podría determinar esto y no solo en estipular lo que sucede cuando se ensamblan dos SSDD. Consideremos, para ilustrar un poco mejor el problema, el caso de $(7)^{6}$. Si suponemos que la sintaxis es "ciega" a todo tipo de información que no sea sintáctica (i.e. configuraciones estructurales y rasgos formales), (5) y (7) deberían ser tratadas de la misma manera. Sin embargo, en este último caso, no resulta obvio cómo derivar el tipo categorial de todo el constituto.<smiles>[Mg]=C[As]</smiles>

Una hipótesis atractiva para explicar la inadecuación del algoritmo de (6) en estos casos consiste en suponer que el ensamble de dos proyecciones máximas produce un objeto sintáctico sin etiqueta, una cláusula mínima de categoría nula, tal y como propone Moro $(1997,2000)$ en su análisis de las estructuras copulativas. Sin embargo, tal alternativa teórica no parece ser correcta para el caso de un análisis simétrico de la aposición explicativa. En el sistema de Moro (2000), la falta de etiqueta en la cláusula mínima predice la posibilidad de extracción de sus constituyentes inmediatos, lo que permite explicar el ascenso de cualquiera de ellos a la posición de sujeto preverbal [Spec, T].

6 Mi intención al introducir dos elementos "abstractos" (sin identificación categorial real) es evitar que nuestro conocimiento "de lingüistas" nos lleve a intentar identificar inductivamente la etiqueta de la construcción. El problema central aquí no es qué categoría corresponde a la estructura de (5), sino qué tipo de mecanismo utiliza la sintaxis para determinarlo. 
(8) Italiano (ejemplo adaptado de Moro 2000: 80)

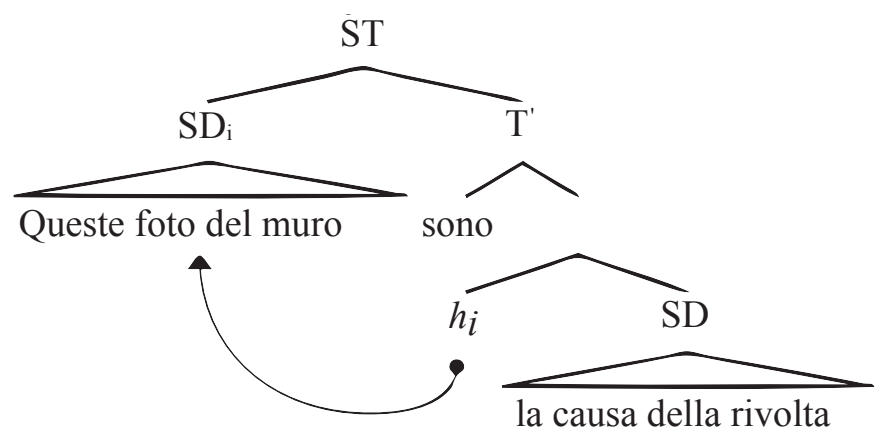

Con respecto a la aposición explicativa, esta predicción resulta completamente incorrecta en un análisis simétrico. Como señala Suñer Grataços (1999: 544), la adyacencia inmediata de ambas partes es un requisito obligatorio de buena formación de la aposición. En términos técnicos, esto indica que no es posible extraer fuera de la construcción apositiva ninguno de sus dos constituyentes inmediatos. Y, sin embargo, la adopción de la hipótesis de etiquetamiento nulo en estructuras simétricas lleva a predecir, bajo supuestos generales, justamente ese fenómeno. En (9), por ejemplo, una categoría T que requiere concordar en persona y número con su sujeto atrae al elemento más cercano en su dominio de mando-c que porte dichos rasgos. En este caso, los elementos que pueden ser atraídos son los constituyentes de la aposición. Esta derivación conduce a oraciones mal formadas como la ejemplificada en (10).

(9)

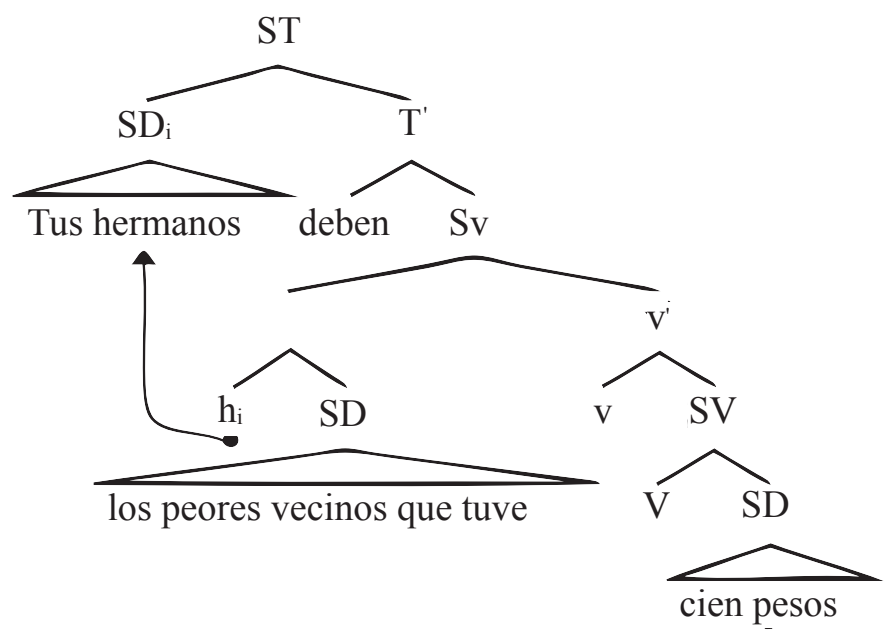


(10) *Tus hermanos deben los peores vecinos que tuve cien pesos.

A partir de esta evidencia, no es posible postular que una aposición explicativa se basa en una cláusula mínima sin etiqueta. Si aún queremos analizar las predicciones que realiza un análisis simétrico de la aposición explicativa, deberemos suponer la existencia de algún tipo de mecanismo que nos permita obtener una etiqueta de tipo $\mathrm{D}$ con rasgos de persona y número a partir de la combinación de dos SSDD. Por suerte, opciones no faltan. Por ejemplo, Chomsky (1994) analiza dos tipos de relaciones de conjuntos a partir de las cuales podríamos obtener el resultado adecuado ${ }^{7}$ : (i) la intersección de los rasgos de ambos $\operatorname{SSDD}(\alpha \cap \beta)$; y (ii) la unión de esos rasgos $(\alpha \cup \beta)$. Dado que el producto de la unión de dos SSDD es potencialmente más complejo que la intersección de rasgos de dos $\mathrm{SSDD}^{8}$, asumiré el mecanismo descrito en (11).

\section{(11) Etiquetamiento vía intersección.}

Si dos objetos $\alpha$ y $\beta$ se ensamblan y ninguno de ellos es un núcleo $\mathrm{X}^{0}$, entonces la etiqueta de la construcción resultante es la proyección de los rasgos que tienen en común $\alpha$ y $\beta$.

Ahora bien, respecto del último punto, debe quedar claro que no estoy proponiendo (11) como parte general de la teoría sintáctica, sino como una simple herramienta que adopto para poder evaluar las predicciones empíricas de un análisis de la construcción apositiva explicativa basado en el ensamble de dos SSDD. De hecho, considero que haber tenido que adoptar este supuesto resulta, desde ya, un enorme defecto para este tipo de análisis: suponemos que, por su distribución sintáctica, la aposición debe ser un SD (u otra categoría que tome sus rasgos), pero, para derivar este resultado a partir de una estructura simétrica, debemos tomar un supuesto sumamente fuerte sobre la naturaleza de las operaciones sintácticas básicas, sin evidencia independiente que lo soporte.

Dado que se asume el modelo de Agree (Chomsky 2001), supongo que los rasgos relevantes para determinar la etiqueta de un ensamble son los

\footnotetext{
7 Si bien Chomsky (1994) descarta a partir de razones conceptuales el etiquetamiento por intersección o unión de conjuntos, su argumento no parece tan convincente a la luz de los supuestos habituales actuales. Véase Boeckx (2008: 85).

8 Por ejemplo, la unión de los rasgos de un $\mathrm{SD}_{\text {Plural }}$ y un $\mathrm{SD}_{\text {Singular }}$ produciría un $\mathrm{SD}$ con los rasgos [+sing] y [+plur]. No es obvio cómo las interfaces podrían lidiar con este tipo de representación sin adoptar otros supuestos.
} 
mismos rasgos que son visibles para dicha operación sintáctica: rasgos- $\varphi$ (persona, género y número) y rasgos categoriales. Así, la combinación de dos SSDD $(D)$ masculinos (masc) de tercera persona (3p) plurales (plur) da como resultado una estructura como la esquematizada en (12).

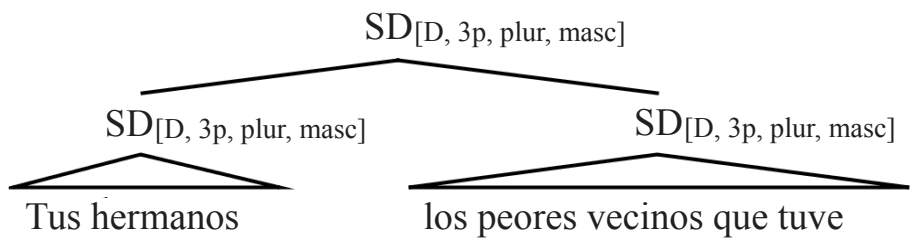

La estructura de (12) permite derivar la condición de adyacencia en la aposición a partir de la condición de A-sobre-A ${ }^{9}$ (Chomsky 1964, Hornstein 2010). Así, en un escenario como el de (13), T atrae a su posición de especificador al elemento más cercano que porte rasgos de persona y número: en este caso, la construcción apositiva en su totalidad.

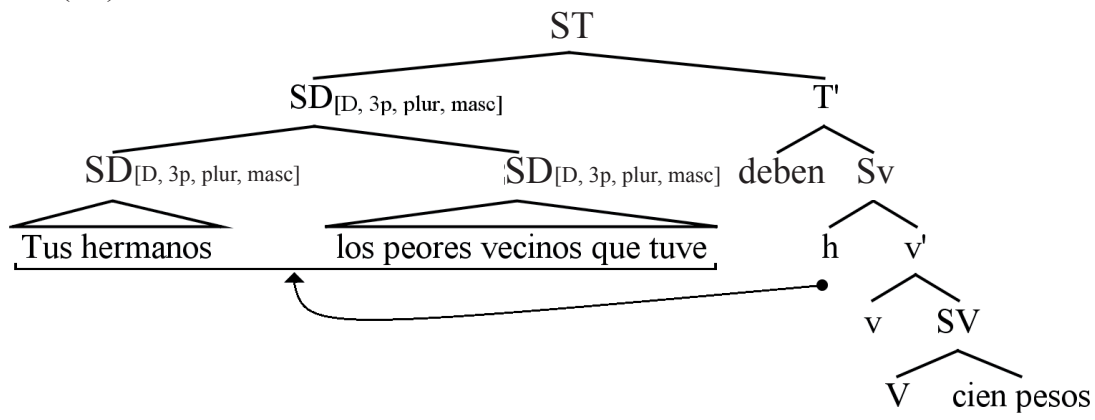

Una ventaja de esta estructura es que explica de manera muy elegante la naturaleza predicativa de la aposición explicativa. Como puede verse en el

9 La versión original de la condición de A-sobre-A dice que si una determinada regla (e.g., movimiento) afecta un elemento A (en el ejemplo, un SD), la regla debe ser aplicada sobre el nodo A más alto. Aquí se utiliza la versión de Hornstein (2010), la cual reduce esta condición a un fenómeno de minimidad. 
par de (14), los significados de una aposición explicativa y de una estructura copulativa son sumamente similares.

(14) a. Tus hermanos, los peores vecinos que tuve, ...

b. Tus hermanos son los peores vecinos que tuve.

Suponer una estructura como la de (12) permite adoptar la base semántica del análisis de Moro (2000) de las construcciones copulativas. Para Moro, la unidad predicativa básica es la cláusula mínima basada en el ensamble de dos proyecciones máximas, dado que esta estructura tiene la posibilidad de capturar la semántica de la predicación en la que "neither the subject nor the predicate prevails" (Moro 2000: 38). En otras palabras, la estructura simétrica representada en (12) permite explicar la relación predicativa que existe entre ambos términos de la aposición ${ }^{10}$.

\section{BOSQUEJANDO UN ANÁLISIS ASIMÉTRICO}

Como recién se discutió, una de las ventajas de adoptar un análisis simétrico de la aposición explicativa es que permite brindar una explicación general a la semántica predicativa de estas estructuras y de las construcciones copulativas. Sin embargo, es necesario señalar que la naturaleza semántica de

10 En una versión previa de este trabajo, se incluía como ventaja del análisis simétrico el hecho de que permitiera que tanto el primero como el segundo término pudieran entrar en relación de concordancia con un elemento externo a la construcción apositiva (un participio, un verbo, etc.). Se seguía, en este aspecto, a Barbeito (2008: 349), quien brinda datos como (iii). El análisis que ella realiza involucra Nosotros como primer término de la aposición y Vialidad, el sector vial como segundo término. Así, el verbo ocupa concordaría exclusivamente con el segundo término de la aposición:

(iii) [[Nosotros], [Vialidad, el sector vial]], ocupa un sector muy importante desde el punto de vista presupuestario en este plan.

Dos observaciones son relevantes con respecto a este tipo de datos. La primera es que las pruebas de juicio gramatical que realicé sobre esta estructura no son consistentes: los hablantes tienden a considerarlas ligeramente agramaticales. La segunda observación es que el Nosotros en esta estructura puede ser mejor analizado como un Tópico Colgado. Evidencia a favor de este análisis es el hecho de que la oración se degrada muchísimo si intentamos ubicar como sujeto postverbal (focalizado informativamente) toda la estructura que Barbeito analiza como aposición.

(iv) *Ocupa [[Nosotros], [Vialidad, el sector vial], un sector muy importante desde el punto de vista presupuestario en este plan. 
la aposición no se restringe solo a la predicación. Como da a entender Potts (2005: 6), la aposición (y las construcciones parentéticas en general) poseen un significado "orientado al hablante" (speaker-oriented). Así, por ejemplo, puede explicarse la clara diferencia que hay en términos pragmáticos entre (15a) y (15b), y la aceptabilidad de (16).

(15) a. \#María dice que Luís es un loco peligroso y que él puede cuidar a los niños.

b. María dice que Luís, un loco peligroso, puede cuidar a los niños.

(16) Federico, este idiota, se olvidó la dirección.

La oración de (15a) es pragmáticamente anómala ya que implica que María sabe que Luis es un loco peligroso y aun así lo considera capaz de cuidar a los niños. Sin embargo, (15b) es pragmáticamente correcta, ya que la frase un loco peligroso se interpreta como una proposición con la que solo el hablante se compromete, no María. Por otra parte, el segundo término de la aposición en (16) puede ser entendido como un comentario de valor subjetivo que refleja la opinión del hablante con respecto a la primera parte de la aposición.

Si bien un análisis que siga lo planteado por Moro (2000) permite explicar la relación predicativa entre los constituyentes de la aposición, los ejemplos de (15) y (16) dan cuenta de un valor semántico-discursivo que no se encuentra presente en las construcciones copulativas.

Una manera de explicar, a la vez, la naturaleza predicativa y discursiva de la aposición es suponiendo que ambos SSDD forman una cláusula mínima sin etiqueta (siguiendo a Moro 2000) que es seleccionada por una categoría funcional que asigna un rol-P (en el sentido de Speas y Tenny $2003^{11}$ ). Por no haber un nombre mejor para esta categoría funcional, la llamaré $A p$ (osición). $A p$, al igual que otras categorías que asignan rasgos interpretativos (como $v)$, requiere recibir rasgos- $\varphi$ a partir de una relación de concordancia. Por tanto, atrae alguno de los SSDD que forman la aposición a su posición de

11 Speas y Tenny (2003) suponen que la interpretación discursiva de ciertos constituyentes puede ser explicada a partir de la asignación de roles-P(ragmáticos), un constructo teórico paralelo a los roles temáticos pero con información puramente pragmática. La existencia de categorías que asignan roles-P estaría basada en la manifestación fonética de estos núcleos en algunas lenguas y en la interpretación especial que reciben sus argumentos. Para el caso de la aposición, ambas condiciones se cumplen (la manifestación fonética de la categoría encargada de introducir el valor discursivo a la aposición sería la entonación prosódica descendente que media entre ambos SSDD). 
especificador. A partir de esta operación, el sintagma que encabeza $A p$ recibe rasgos- $\varphi$. La estructura generada hasta este punto se ilustra en (17).

(17)

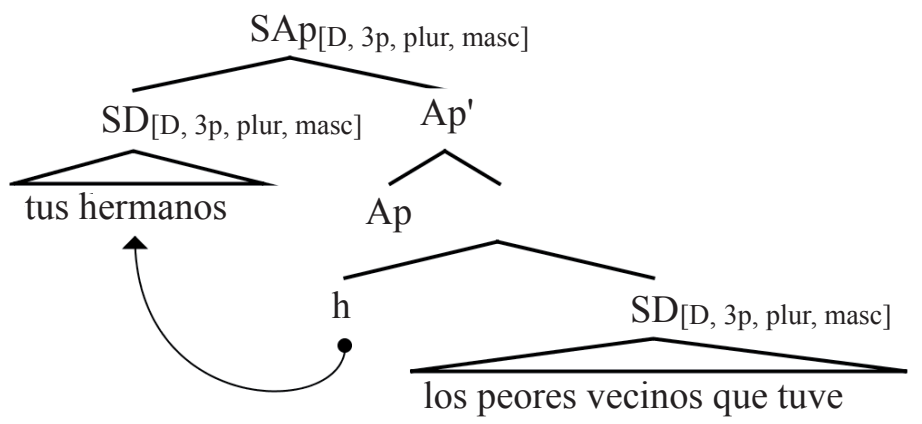

La caracterización que propongo para la categoría $A p$ es, salvando las distancias, sumamente similar a la que Rizzi (1997: 285) da para la categoría Tópico: el especificador de $A p$ es un elemento prepuesto al resto de la cláusula mínima y separado prosódicamente de ella a partir de una "entonación de coma". El remanente de la cláusula mínima (el segundo término de la aposición) funciona como un comentario desde la perspectiva del hablante sobre el elemento prepuesto.

Este análisis asimétrico, al igual que el propuesto a partir de relaciones de mando-c mutuo en el apartado anterior, deriva de la condición de adyacencia en la aposición como un corolario del principio de A-sobre-A. Así, por ejemplo, si T busca en su dominio de mando-c una categoría con rasgos de número y persona, el elemento más cercano que encontrará es el sintagma apositivo $^{12}$. Por el escenario de minimidad que plantearía, T no puede atraer ninguno de los términos de la aposición.

12 Esto puede ser problemático para postulaciones del EPP en las que éste se plantea como requerimiento de un constituyente $[+\mathrm{D}]$ en la posición de especificador de la categoría atractora. Siguiendo a Pesetsky y Torrego (2001), asumo que el EPP es "un rasgo de un rasgo". En otras palabras, el movimiento a la posición de especificador de una categoría no se basa en la necesidad de un elemento $[+D]$, sino en un requisito estructural de alguno de los rasgos que establecen concordancia. Dejo abierto el tema de cuál de ellos puede ser para el caso de la aposición. 


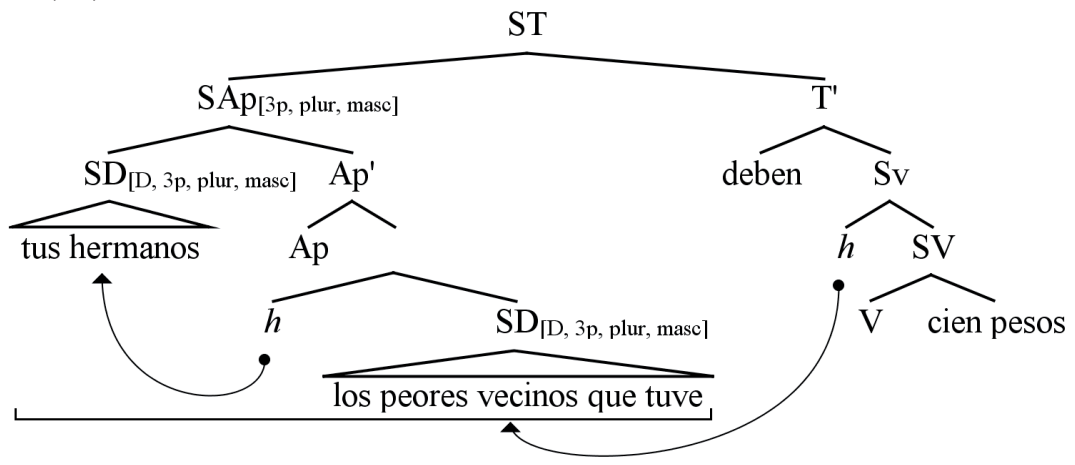

Una de las propiedades particulares de la aposición explicativa que debe ser derivada a partir de nuestro análisis es la posible presencia de adjuntos adverbiales antepuestos al segundo término de la aposición (19a), pero no al primero (19b).

(19) a. [Tus hermanos, seguramente los peores vecinos que tuve], deben cien pesos.

b. *[Seguramente tus hermanos, los peores vecinos que tuve], deben cien $\operatorname{pesos}^{13}$.

('Tus hermanos seguramente son los peores vecinos que tuve $y$ deben cien pesos').

Es importante recordar que la mayoría de los adjuntos adverbiales no pueden ubicarse dentro de un SD (Ernst 2002: 8).

(20) a. [ ${ }_{\mathrm{SD}} *$ Seguramente tus hermanos]

b. $\left[{ }_{\mathrm{SD}} *\right.$ Seguramente los peores vecinos que tuve $]$

La interpretación del sujeto de (19a), en donde el adverbio tiene alcance sobre la predicación, y la restricción ilustrada en (20) llevarían a suponer que el adjunto modifica la cláusula mínima que contiene ambos términos

13 Es importante no confundir (19b) con una estructura en la que el adverbio se ubica en la periferia izquierda:

(vii) Seguramente, [tus hermanos, los peores vecinos que tuve], deben cien pesos.

('Tus hermanos son los peores vecinos que tuve y seguramente deben cien pesos'). 
de la aposición. No obstante, esta idea debería ser descartada en un análisis simétrico como el propuesto en el apartado anterior, dado que una estructura como (21) prediría el orden de palabras incorrecto (es decir, el ejemplificado en $(19 b))^{14}$.

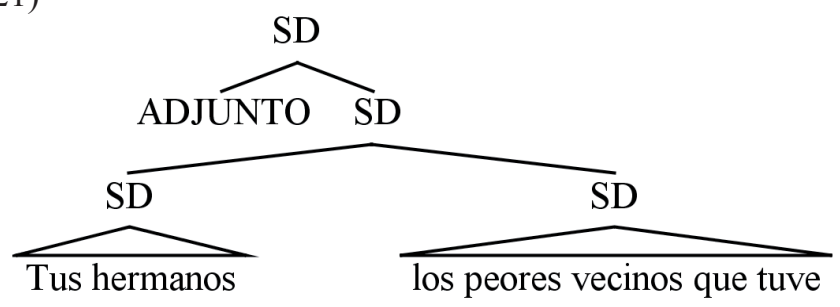

Sin embargo, el análisis asimétrico basado en el ascenso de uno de los términos de la aposición a la posición de especificador de una categoría discursiva permite, a la vez, explicar tanto la semántica como el orden correcto de una oración como (19a).

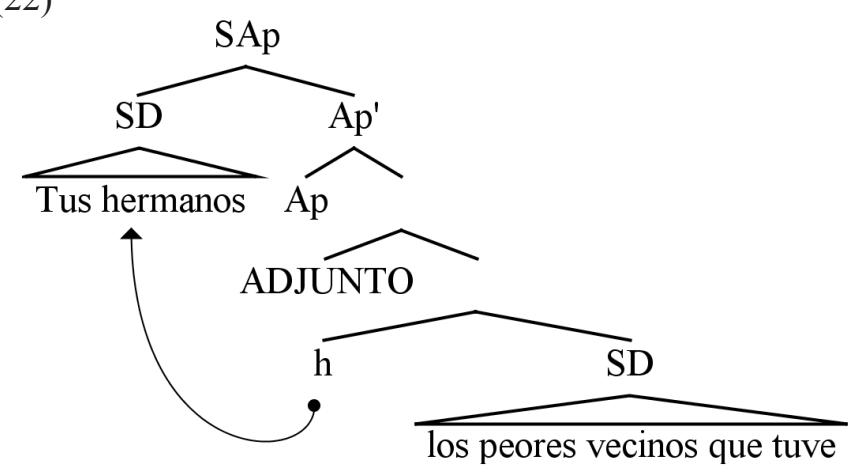

Otra ventaja del análisis asimétrico es que permite explicar el dispar comportamiento que existe entre ambas partes de la aposición en términos

14 Si bien no es posible adjuntar adverbios a SSDD, no es claro si esta restricción se sostiene para un constituyente con etiqueta SD derivada a partir del mecanismo de (11). Dicha restricción bien podría ser de naturaleza semántica (e.g., los adverbios solo modifican eventos, por lo que se adjuntan solo a proyecciones verbales y funcionales) y no de una restricción puramente categorial (basada en etiquetas). 
de ligamiento de variables. Es generalmente aceptado que un cuantificador debe mandar-c la variable que liga. Una derivación como las propuestas en (18) o (22) predice que un cuantificador en el primer término de la aposición debe ligar siempre a una variable en el segundo término, y que el caso inverso no puede darse. Como ilustran los ejemplos de (23) y (24), este patrón se cumple.

(23) a. Todo ciudadano, un individuo con derechos y obligaciones, debería pagar los impuestos.

b. ?? Un individuo con derechos y obligaciones, todo ciudadano, debería pagar los impuestos.

(24) a. Algunas personas, dos de los aquí presentes, conocen la verdad.

b. *Dos de los aquí presentes, algunas personas, conocen la verdad.

Un análisis simétrico, en cambio, predice la buena formación de (23b) y (24b), ya que involucra una relación de mando-c mutuo entre ambos términos de la aposición.

Más allá de su mayor alcance empírico, el análisis asimétrico no requiere la postulación de ningún tipo de principio gramatical independiente (como el Etiquetamiento vía Intersección de (11)). Esto lo convierte en un tipo de descripción de la aposición explicativa mucho más simple que el habitualmente utilizado (basado en la propuesta de Hockett (1958)).

\section{CONCLUSIÓN}

En el presente trabajo se han evaluado los requisitos teóricos y la adecuación empírica de la descripción que típicamente se asume para la aposición explicativa. Ésta se basa en la idea de Hockett (1958) de que existe una relación de simetría entre ambas partes de la aposición. Sin embargo, se ha visto que la real implementación de esta idea requiere la postulación de principios especiales con respecto a las operaciones básicas de la gramática. Como alternativa, se propone un análisis basado en una relación simétrica que luego se rompe a partir del ascenso de uno de los constituyentes de la aposición a una posición sintáctica que fundamenta la especial interpretación discursiva de la construcción. Este análisis, se ha demostrado, siendo teóricamente más simple, provee a la vez una mayor capacidad predictiva con respecto a las propiedades de la aposición explicativa. 


\section{REFERENCIAS BIBLIOGRÁFICAS}

Barbeito, Vanina. 2008. Hablemos de eso, la aposición. En Cristina Messineo, Marisa Malvestitti y Roberto Bein (eds.). Estudios de lingüistica y Antropología. Homenaje a Ana Gerzenstein. Buenos Aires: Facultad de Filosofía y Letras, UBA.

Boeckx, Cedric. 2008. Bare Syntax. Oxford: Oxford University Press.

Сномsкy, Noam. 1964. Current issues in linguistic theory. The Hague: Mouton.

1994. Bare phrase structure. En MIT Occasional Papers in Linguistics 5. Cambridge, MIT. 1995. El Programa Minimalista. Madrid: Alianza.

2001. Derivation by phase. En Kenstowicz, M. (ed.). Ken Hale: A life in language, Cambridge, MA: MIT Press.

2008. On Phases. En Robert Freidin, Carlos Otero y María Luisa Zubizarreta (eds.). Foundational Issues in Linguistic Theory, pp. 133-166. Cambridge, MA: MIT Press.

Di Tullio, Ángela. 1997. Manual de Gramática del Español. Buenos Aires: Edicial.

Ernst, Thomas Boyden. 2002. The Syntax of Adjuncts. Cambridge: Cambridge University Press.

Hockett, Charles. 1958. Curso de Lingüística Moderna. Buenos Aires: Eudeba.

HornsteIn, Norbert. 2010. A Theory of Syntax: minimal operations and universal grammar. Cambridge, MA: Cambridge University Press.

Kovacci, Ofelia. 1990. El comentario grammatical: teoría y práctica (I). Madrid: Arco Libros.

Moro, Andrea. 1997. Dynamic Antisymmetry: Movement as a symmetry-breaking phenomenon. Studia Linguistica 51: 50-76. 2000. Dynamic Antisymmetry: Movement as a Symmetry-breaking Phenomenon. Cambridge, MA: MIT Press.

Pesetsky, David y Esther Torrego. 2001. T-to-C movement: Causes and consequences. En Michael Kenstowicz (ed.). Ken Hale: A life in language, pp. 355-426. Cambridge, MA: MIT Press.

PotTs, Chris. 2005. The logic of conventional implicatures. New York: Oxford University Press.

Rizzi, L. 1997. The fine structure of the left periphery. En Liliane Haegeman (ed.). Elements of grammar, pp. 281-337. Dordrecht: Kluwer.

Speas, Peggy y Carol Tenny. 2003. Configurational Properties of Point of View Roles. En Anna-Maria DiSciullo (ed.). Asymmetry in Grammar, pp. 315-344. Amsterdam: John Benjamins.

Suñer Grataços, Avellina. 1999. La aposición y otras relaciones de predicación en el sintagma nominal. En Ignacio Bosque y Violeta Demonte (eds.). Gramática Descriptiva de la Lengua Española, vol I, pp. 523-564. Madrid: Espasa. 